\title{
Bi-allelic PAGR1 variants are associated with microcephaly and a severe neurodevelopmental disorder: Genetic evidence from two families
}

\author{
Hagit Daum ${ }^{1 \dagger}$ (1) | Mythily Ganapathi ${ }^{2 \dagger}$ | Yoel Hirsch ${ }^{3}$ | Emily L. Griffin ${ }^{4}$ | \\ Charles A. LeDuc ${ }^{4}$ | Jacob Hagen ${ }^{4}$ | Simcha Yagel ${ }^{5}$ | Vardiella Meiner ${ }^{1}$ | \\ Wendy K. Chung ${ }^{6 \neq}$ | Hagar Mor-Shaked ${ }^{1 \ddagger}$ \\ ${ }^{1}$ Department of Genetics, Hadassah Medical Organization and Faculty of Medicine, Hebrew University of Jerusalem, Israel \\ ${ }^{2}$ Department of Pathology \& Cell Biology, Columbia University, New York, New York, USA \\ ${ }^{3}$ Dor Yeshorim, Committee for Prevention of Jewish Genetic Diseases, Brooklyn, New York, USA \\ ${ }^{4}$ Department of Pediatrics, Columbia University, New York, New York, USA \\ ${ }^{5}$ Obstetrics and gynecology, Hadassah Medical Organization and Faculty of Medicine, Hebrew University of Jerusalem, Israel \\ ${ }^{6}$ Departments of Pediatrics and Medicine, Columbia University, New York, New York, USA
}

\section{Correspondence}

Hagit Daum, MD, Department of Genetics, Hadassah-Hebrew University Medical Center, POB 12000, Jerusalem, Israel 9112001.

Email: dhagit100@gmail.com

Wendy Chung, MD PhD, Kennedy Family Professor of Pediatrics and Medicine, Chief, Clinical Genetics, Columbia University, 1150 St. Nicholas Avenue, Room 620, New York, NY 10032.

Email: wkc15@cumc.columbia.edu

\section{Funding information}

National Human Genome Research Institute grant, Grant/Award Number: R01 HG009141; the National Heart, Lung and Blood Institute grant, Grant/Award Number: UM1 HG008900; National Human Genome Research Institute; National Eye Institute; National Human Genome Research Institute; Broad Institute; JPB Foundation

\begin{abstract}
Exome and genome sequencing were used to identify the genetic etiology of a severe neurodevelopmental disorder in two unrelated Ashkenazi Jewish families with three affected individuals. The clinical findings included a prenatal presentation of microcephaly, polyhydramnios and clenched hands while postnatal findings included microcephaly, severe developmental delay, dysmorphism, neurologic deficits, and death in infancy. A shared rare homozygous, missense variant (c.274A > G; p.Ser92Gly, NM_024516.4) was identified in PAGR1, a gene currently not associated with a Mendelian disease. PAGR1 encodes a component of the histone methyltransferase MLL2/ MLL3 complex and may function in the DNA damage response pathway. Complete knockout of the murine Pagr1a is embryonic-lethal. Given the available evidence, PAGR1 is a strong candidate gene for a novel autosomal recessive severe syndromic neurodevelopmental disorder.
\end{abstract}

KEYWORDS

Ashkenazi Jews, infantile-lethal disorder, microcephaly, neurodevelopmental disorder, PAGR1

\section{1 | INTRODUCTION}

PAGR1 encodes for PAXIP1-associated glutamate-rich protein 1, which is a component of a Set1-like multiprotein histone methyltransferase complex (Kumar et al., 2014). Its roles remain elusive with diverse

${ }^{\dagger}$ These authors should be considered joint first authors.

* These authors should be considered joint senior authors. proposed biological mechanisms of action. It was first identified in a complex with PAX-Interacting Protein 1 (PAXIP1; also known as PTIP) by co-immunoprecipitation and mass spectrometry (Cho et al., 2007). Both proteins were found to be associated with MLL3/MLL4-containing histone $\mathrm{H} 3 \mathrm{~K} 4$ methyltransferase complexes, suggesting a role in epigenetic gene regulation. In addition, PAGR1 and PAXIP1 form a separate complex that plays a role in DNA repair (Gong et al., 2009). PAGR1 also acts as a transcriptional co-regulator of the estrogen and glucocorticoid 
receptors (Liang et al., 2009; Zhang et al., 2013), a function thought to be independent of its association with PAXIP1 or MLL3/MLL4.

While the biological functions of this protein are obscure, studies in animal models suggest this gene plays a key role in early development. In zebrafish, loss of function of this gene is associated with reduced brain ventricle size, and less distinctly defined midbrain-hindbrain boundary (Blaker-Lee et al., 2012). Null mice do not survive beyond early stages of embryogenesis (four to five somite). They have abnormal development of extra-embryonic tissues including the amnion, chorion and visceral yolk sac. At the molecular level, Pagr1a null embryos have reduced expression of Bmp2, a known regulator of extraembryonic development, and reduced expression of the forebrain marker Otx2 and Six3 during neural development (Kumar and others 2014). In addition, a recent study demonstrated that deletion of Pagr1 in pre-adipocytes prevents the induction of $\mathrm{C} / \mathrm{EBP} \beta$ and $\mathrm{C} / \mathrm{EBP} \delta$ and disrupts adipogenesis and muscle development (Lee et al., 2020).

To date, PAGR1 has not been associated with any Mendelian disorder. Here we present details of three individuals from two unrelated families of Ashkenazi Jewish (AJ) ancestry, harboring the same homozygous missense variant in the PAGR1 gene, who demonstrated a clinically similar neurodevelopmental phenotype.

\section{2 | METHODS}

\subsection{Exome sequencing}

Family A: Following written informed consent and IRB approval, exome analysis was performed on DNA extracted from amniocentesis of the proband (female fetus) and parents in family $A$ (trio exome). Exonic sequences from genomic DNA were enriched with the SureSelect Human All Exon 50 Mb V5 Kit (Agilent Technologies, Santa Clara, California, USA). Sequences were generated on a HiSeq2500 sequencing system (Illumina, San Diego, California, USA) with 125 bp paired-end runs. Read alignment and variant calling were performed with DNAnexus (Palo Alto, California, USA) using default parameters with the human genome assembly hg19 (GRCh37) as reference. Filtering was performed as described elsewhere (Abu-Libdeh et al., 2021) Exome analysis of the proband yielded 53 million reads, with a mean coverage of $78 \mathrm{X}$, and $94 \%$ over $20 \mathrm{X}$.

Family B: Clinical exome sequence analysis of the two affected siblings (males) and their parents (quad exome) done in 2014 was non-diagnostic. The details of the methodologies were previously described (Retterer et al., 2016).

\subsection{Whole Genome sequencing}

For family A, next generation sequencing (NGS) libraries were prepared with an Illumina PCR-free TruSeq DNA Library Prep Kit, from the proband's genomic DNA. Sequences were generated on an Illumina NovaSeq 6000 sequencing platform as 150 bp paired-end reads, to a final depth of 30X coverage. The FASTQs were uploaded into the Geneyx (previously TGex) Analysis platform (Dahary et al., 2019). Alignment and variant calling of single nucleotide variants (SNVs), copy number variants (CNVs) and structural variants (SVs), and repeats were called using Illumina DRAGEN Bio-IT. The resulting VCF file were comprehensively annotated on the Geneyx Analysis annotation engine, and presented for analysis, filtering and interpretation. Variant prioritization was performed using VarElect (Stelzer et al., 2016).

Written informed consents were obtained for family B and research whole genome sequencing was performed on the peripheral blood DNA samples from the two affected siblings and the unaffected parents. Sequencing methods and variant filtering criteria were previously described (Okur et al., 2019). The candidate variants and their familial segregation with the clinical phenotype were confirmed by Sanger sequencing.

\section{3 | Segregation analysis}

The region containing the PAGR1 variant was amplified by PCR using the genomic DNA from the members of the two families and analyzed by Sanger dideoxy nucleotide sequencing.

\section{4 | Haplotype analysis}

Using family $\mathrm{B}^{\prime} \mathrm{s}$ genome sequencing data, a $3 \mathrm{Mb}$ region (Chr16:27816799-31,816,799, hg38) centered on the PAGR1 variant was queried for variants ( $G Q>30$, variant allele fraction $>0.2$ ). Almost all the variants in this region were homozygous in both affected individuals in family B indicating a stretch of homozygosity, and the parents were heterozygous at these positions (Supplemental Table 2). The results were compared with the variants in the proband from family $A$ and homozygous stretches common to the affected individuals of the two families were identified.

\section{5 | Carrier frequency analysis}

31,513 anonymous blood samples were obtained from the Dor Yeshorim screening program, from around the world (including the United States of America [New York, New Jersey, Maryland, California, Illinois, Florida, Ohio, and Michigan], Canada, Mexico, Argentina, Brazil, UK, Belgium, France, Switzerland, Austria, Australia, South Africa, and Israel) (Ekstein and Katzenstein 2001). All participants provided written consent for research. The consent included that the participant samples would be used for clinical testing and residual material would be de-identified to use for research purposes to characterize single gene disorders in the Ashkenazi Jewish population. 


\section{3 | RESULTS}

\section{1 | Clinical Reports}

The clinical features seen in the three affected individuals from the two families are listed in Table 1.

Family A. A healthy non consanguineous couple of Ashkenazi Jewish (AJ) descent, with one healthy child, was referred for genetic counseling during their second pregnancy because of sonographic findings in the third trimester including moderate polyhydramnios, clenched hands, and microcephaly (head circumference 0.6th centile, cerebellum 2-3rd centile) (Figure 1A, individual II-2). The pregnancy was relatively normal initially with nuchal translucency of $1.1 \mathrm{~mm}$ and a low integrated risk for trisomy $21(1: 20,000)$ followed by a normal second trimester anatomy scan. However, at the 24th week there was a small head (head circumference 1st centile) with presumed normal corpus callosum, vermis and cerebellum.

The pregnancy was terminated at week 32. Post-mortem examination showed a sloping forehead, micrognathia and clenched hands (Figure 1B, panel 1).
Family B. The two affected individuals were brothers, sons of a consanguineous healthy Ashkenazi Jewish couple. The proband (Figure 1A, II-2) presented at birth with multiple congenital anomalies including hydrocephalus, low-lying conus medullaris, coarctation of the aorta, and flexion contractures (Figure 1B, panel 2). The pregnancy, including anatomy scan, was reportedly normal. The baby was delivered at 40 weeks and 2 days of gestation with Apgar scores of 9 and 9 at 1 and 5 minutes. His birth weight was 2055 grams $(<5$ th centile), length was $46.5 \mathrm{~cm}$ ( $\sim 5$ th centile), and head circumference was $26.5 \mathrm{~cm}$ ( $<<5$ th centile). He was noted at birth to have normal tone but a weak suck. Dysmorphic features were noted, including microcephaly with a sloping forehead and overriding sutures, hypotelorism, large, low-set ears posteriorly rotated, bulbous nose with broad nasal bridge, high arched palate, short neck with excess skin folds, wide spaced nipples, bilateral simian crease, overlapping fingers with hypoplastic nails, a sacral dimple, cryptorchidism, inverted and medially rotated feet, and overlapping of toes.

He was admitted to the neonatal intensive care unit for medical management and was found to be hypertonic. Spinal ultrasound demonstrated a low-lying conus medullaris. Chest $\mathrm{x}$-ray demonstrated

TABLE 1 Clinical features of the three affected individuals harboring homozygous variant in PAGR1

\begin{tabular}{|c|c|c|c|}
\hline & Individual 1 (Family A) & Individual 2 (Family B) & Individual 3 (Family B) \\
\hline Parental ancestry & \multicolumn{3}{|l|}{ Ashkenazi Jewish } \\
\hline Consanguinity & No & Yes & \\
\hline Family history & None & None & Affected brother \\
\hline Gravida, Parity & G2P1 & G2P1 & G3P2 \\
\hline Spontaneous $(\mathrm{Y} / \mathrm{N})$ & $\mathrm{Y}$ & $\mathrm{Y}$ & $\mathrm{Y}$ \\
\hline \multicolumn{4}{|l|}{ Pregnancy follow-up } \\
\hline $\begin{array}{l}\text { First trimester: } \\
\text { Nuchal translucency }\end{array}$ & $1.1 \mathrm{~mm}$ & NA & Thick \\
\hline Integrated test & $1: 20,000$ & NA & NA \\
\hline $\begin{array}{l}\text { Second trimester: } \\
\text { First anomaly scan (15th } \\
\quad \text { week) }\end{array}$ & $\begin{array}{l}\text { Normal (left ventricular } \\
\text { echogenic focus) }\end{array}$ & NA & NA \\
\hline Second anomaly scan & $\begin{array}{l}\text { Normal CC and vermis. } \\
\text { Cerebellum } 8 \%\end{array}$ & Reported normal & Small for gestational age \\
\hline 24th week & \multicolumn{2}{|l|}{$\begin{array}{l}\text { HC } 1 \%, \text { normal CC, vermis \& } \\
\text { cerebellum } 8 \%\end{array}$} & Intrauterine growth restriction \\
\hline Pregnancy outcome & Termination of pregnancy & A delivery of a baby boy at $40+2$ weeks & A delivery of a baby boy at $39+2$ weeks \\
\hline Birth weight & NA & 2055 gr (<5\%ile) & 2450 gr (3-5\%ile) \\
\hline Apgar score & NA & $9 / 9$ & $8 / 9$ \\
\hline Birth HC (\%) & NA & $26.5 \mathrm{~cm}(<<5 \%$ ile $)$ & $31 \mathrm{~cm}$ (<5\%ile) \\
\hline Clinical description & \multicolumn{3}{|c|}{ Dysmorphic features as detailed in text } \\
\hline Additional findings & NA & \multicolumn{2}{|l|}{ Aortic coarctation with PDA } \\
\hline Outcome & NA & $\begin{array}{l}\text { Died at } 10 \text { months of age due to cardiac } \\
\text { arrest following a surgery }\end{array}$ & $\begin{array}{l}\text { Died at } 3 \text { months of age of cardiac arrest } \\
\mathrm{m} / \mathrm{p} \mathrm{d} / \mathrm{t} \text { to aspiration }\end{array}$ \\
\hline Development & NA & \multicolumn{2}{|l|}{ No milestones } \\
\hline CMA & Normal & Normal, long stretches of $\mathrm{ROH}$ & Not performed \\
\hline $\begin{array}{l}\text { Exome/Genome } \\
\text { sequencing [hg19] }\end{array}$ & \multicolumn{3}{|c|}{$\begin{array}{l}\text { PAGR1 homozygous variant } \\
\text { chr16:29828120-A-G; } \\
\text { NM_024516.4: c.[274A > G];[274A > G] } \\
\text { NP_078792.1: p.[(Ser92Gly)];[(Ser92Gly)] }\end{array}$} \\
\hline
\end{tabular}

Abbreviations: CC - corpus callosum, CMA - chromosomal microarray analysis, $d / t$ - due to, HC - head circumference, HMZ - homozygous, m/p - most probably, NA - not available, ROH-region of homozygosity. 
(a) Family A

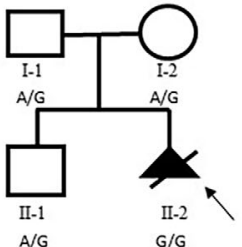

(b)

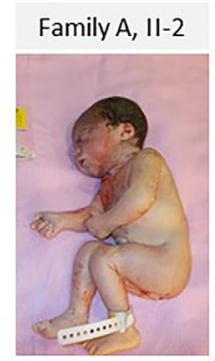

Panel 1
Family B

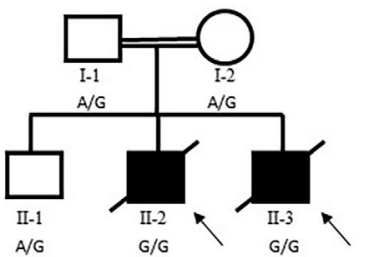

A/G

(c)

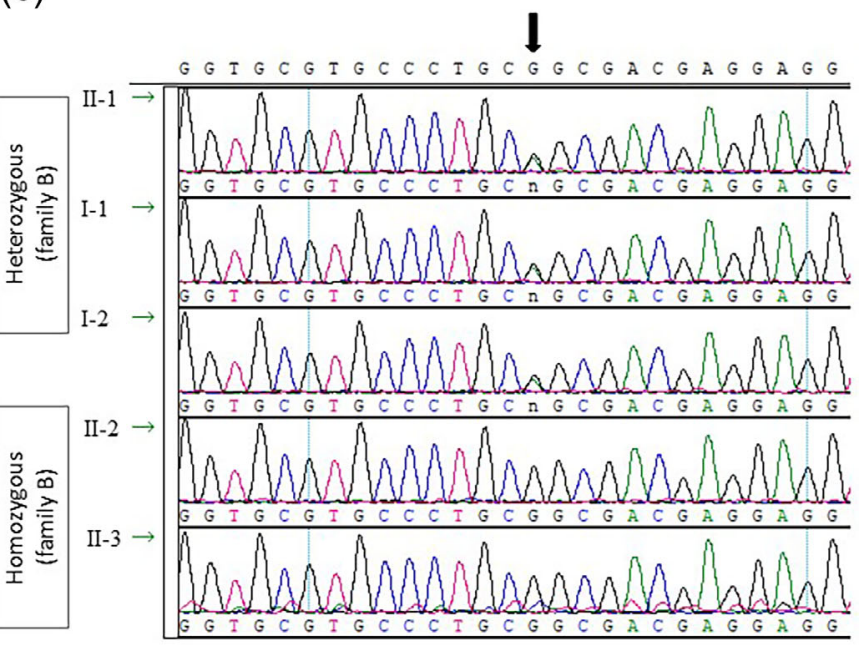

FIGURE 1 Molecular genetics and pictures of the affected individuals. 1a. Pedigrees of both Ashkenazi Jewish families. 1b. Pictures of the three affected individuals; the terminated fetus of family A and two affected males of family B. Notice the resemblance of the clenched hands, small chin and sloping forehead related to microcephaly. 1c. Sanger sequencing results for the PAGR1 variant in the members of family B

thin, gracile ribs. Echocardiogram at two days of age demonstrated aortic coarctation, a large patent ductus arteriosus (PDA), and a patent foramen ovale with bidirectional flow; follow-up echocardiogram four days later demonstrated that both the coarctation and PDA had resolved. Renal ultrasound was normal. Head ultrasound on the second day of life was normal. Brain MRI on day of life six was normal. Electroencephalogram (EEG) on day 22nd of life demonstrated subclinical seizures, and he was started on phenobarbital. Repeat EEG two days later was negative for seizure activity. He was noted to have aphonia on his sixth day of life. Laryngoscopy was inconclusive. $\mathrm{He}$ had a G-tube placed at one month of age as he tolerated minimal oral intake. His course was complicated by multiple episodes of central apnea and chronic respiratory failure for which a tracheotomy tube was placed at two months of age. He was transferred to a rehabilitation facility and was not found to meet any developmental milestones. At 10 months of age, he was taken to surgery due to an infection at the gastrostomy-tube site. He had a cardiac arrest following surgery and died. Genetic evaluation included a fluorescent in-situ hibridization analysis for chromosomes $13,18,21 \mathrm{X}$, and $\mathrm{Y}$, which was normal, and a chromosome microarray analysis which showed long regions of homozygosity consistent with the parental consanguinity.

The brother of the proband presented prenatally with similar features (Figure 1A, B, II-3). An increased nuchal fold was noted on a 15-week ultrasound. He was reported at 20 weeks gestation to be small for gestational age and at 24 weeks of gestation was found to have intrauterine growth restriction. At 30 weeks of gestation, he was found to have microcephaly, low set ears, echogenic bowel, possible anal atresia, clenched hands, and sluggish fetal movement. He was delivered at 39 weeks 2 days gestation with Apgar scores of 8 and 9 at 1 and 5 minutes, respectively. His birth weight was 2450 grams ( $3-5$ th centile), length $45.5 \mathrm{~cm}$ (3-5th centile), and head circumference of $31 \mathrm{~cm}(<<5$ th centile). At birth, he was noted to have normal tone but a weak suck. He had dysmorphic features including microcephaly with sloping forehead, small palpebral fissures, large, low-set ears with over-folded prominent lobes, bulbous nose, high arched palate, retrognathia, short neck, wide spaced nipples, bilateral undescended testicles, and positional bilateral talipes equinovarus.

He was admitted to the neonatal intensive care unit for medical management. He was found to have hypertonia and a failed his hearing screen. Head ultrasound on his first day of life demonstrated decreased sulcal patterning suggestive of prematurity, though he was full term. Brain MRI on his fifth day of life demonstrated a punctate focus of restricted diffusion in the brain parenchyma suggestive of a small acute infarction; there were no gross structural abnormalities. EEG was negative for seizure activity. Echocardiogram showed a small atrial septal defect versus patent foramen ovale. Renal ultrasound was normal. Spinal ultrasound was normal and did not demonstrate evidence of a tethered cord. He was discharged to rehabilitation on nasogastric tube feeding. He was reported not to achieve any developmental milestones. He died at 3 months of age of cardiac arrest presumed to be secondary to aspirations. Clinical quad exome sequencing (both affected siblings and the parents) originally reported in June 2014 was non-diagnostic.

\section{2 | Identification of a homozygous variant in PAGR1}

For family A, amniocentesis was performed at the 28th week, prior to termination. Chromosomal microarray analysis showed normal female 
karyotype and trio-exome sequencing analysis revealed a single homozygous missense variant, in PAGR1 (chr16:29828120-A-G, hg19, NM_024516:c.274A>G, p.Ser92Gly, rs375215655). Sanger sequencing (Figure $1 C$ ) revealed both parents and the healthy sibling to be heterozygous carriers. This variant was present within a relatively small region of homozygosity on chromosome 16 (Chr16:29-31.4 Mb, hg19). In order to comprehensively assess all the possible genetic etiologies, whole genome sequencing of the proband from family $\mathrm{A}$ (Figure 1A, II-2) was performed. The analysis, which included SNVs, CNVs and SVs did not reveal any other candidates apart from the homozygous PAGR1 variant. A focused analysis of the homozygous block encompassing the PAGR1 variant (chr16:29,725,143-31,470,540, hg 19) did not reveal any other rare variants in the coding region, nor any suspected copy/structural variants.

For family $\mathrm{B}$, whole genome sequencing analysis of the affected sibs identified candidate rare variants including homozygous missense changes in PAGR1, KDM8, and SLC12A3 and a hemizygous variant in PRPS2 (Supplemental Table 1). The SLC12A3 variant was deprioritized based on the phenotypic differences when compared to the clinical features associated with this gene (Gitelman syndrome [MIM 263800]). We initially focused on PRPS2 and PAGR1. The PRPS2 variant was present in the unaffected brother (data not shown) which resulted in eliminating this candidate. Segregation of the PAGR1 variant in the unaffected sibling (II-1; Figure 1C) and phenotype comparisons resulting from Genematcher matches (Sobreira et al., 2015), led to the prioritization of the PAGR1 homozygous missense variant (chr16:29828120-A-G, hg19, NM_024516.4:c.274A > G, p.Ser92Gly) as the strongest candidate for the phenotype in the affected siblings. Notably, this variant was present within a region of homozygosity on chromosome 16 (Chr16:19.29-85.94 Mb, hg19) as seen in the chromosomal microarray results for the individual II-2.

The PAGR1 c.274A > G; p.Ser92Gly variant is present at a very low allele frequency in GnomAD v2 (allele frequency 0.00008307, 17/204636 heterozygotes) and v3 (allele frequency $0.00008544,13 / 152158$ heterozygotes) databases with no homozygous individuals Upon clinical presentation it was deposited in the ClinVar database as a likely pathogenic variant (accession number SCV001733594.1). This variant is in the conserved PAXIP1_C domain of the protein and has the following in silico prediction scores Provean -3.48 (damaging), SIFT O (damaging), REVEL 0.2119 (benign), MetaSVM -0.5887 (tolerated), CADD (GRCh38-v1.6) 24.8 and GERP 5.34. The Serine 92 amino acid residue is conserved in organisms including Xenopus, zebrafish, mouse, dog and elephant.

Analysis of SNP genotypes in a $3 \mathrm{Mb}$ region flanking the PAGR1 variant showed shared homozygous variants in a sub-region (chr16:2978 6327-30,169,169 [hg38], 382Kb), in the three affected individuals from the two families (family A II-2, family B II-2, II-3) indicating a shared contiguous stretch of homozygosity (Supplemental Tables 2 and 3).

\section{3 | Carrier frequency of the PAGR1 variant in Ashkenazi Jews}

To determine the carrier frequency of the PAGR1 variant in the Jewish population, 31,513 individuals were screened (22,909 fully Ashkenazi
Jewish, 4813 Sephardi Jewish, and 3791 mixed Ashkenazi Jewish/ Sephardi Jewish). Out of the 22,909 fully Ashkenazi samples, fiftyeight were heterozygous, with a carrier frequency of 1/394 (an allele frequency of 0.0012). Out of the 3791 Ashkenazi/Sephardi individuals, one sample was heterozygous. There were no carriers within the 4813 individuals of Sephardi Jewish descent. Homozygous individuals for the PAGR1 variant were not detected in any of the cohorts.

\section{4 | DISCUSSION}

We describe three affected individuals from two unrelated Ashkenazi Jewish families with a homozygous missense variant in PAGR1 (c.274A>G; p.Ser92Gly; rs375215655). The phenotypes of the affected individuals from both families are similar and include microcephaly, distal arthrogryposis with clenched hands, and micrognathia.

PAGR1 maps to the 16p11.2 recurrent region (BP4-BP5) wherein microdeletions and microduplications are associated with a broad range of neurodevelopmental impairments, including autism, intellectual disability, language disorders, and sensory symptoms (Al-Jawahiri et al., 2019; Jenkins et al., 2016; Kim et al., 2020; Niarchou et al., 2019; Shinawi et al., 2010; Steinman et al., 2016) (MIM 611913, 614,671). Several studies have examined the role of PAGR1 in the neurocognitive phenotype of 16p11.2 copy number variants, including a recent one on fetal cortical neurogenesis (Morson et al., 2021). Review of the microarray-based "BrainCloud" dorsolateral prefrontal cortex transcriptome found PAGR1 to be ubiquitously expressed with predominant expression in the cerebellum, and its expression is significantly enriched in the fetal period as compared to the postnatal period (Birnbaum et al., 2014).

While not much is known about PAGR1 and its function in humans, several studies in model organisms suggest its clinical importance. In zebrafish, reduced expression of PAGR1 (referred as c16orf53) was associated with reduced brain ventricle size, in addition to less sharply defined midbrain-hindbrain boundaries (Blaker-Lee et al., 2012). A recent study in mice revealed that expression of the cognate mouse gene Pagr1a is found predominantly in the extraembryonic and chorionic ectoderm from pre-gastrulation stages and shows high expression within the embryo. In addition, homozygous knock out mice are non-viable and do not develop beyond the fourto five-somite stage (Lee et al., 2020).

However, so far, there are no human Mendelian phenotypes associated with the bi-allelic disruption of PAGR1. Assessing the probability of PAGR1 causing an autosomal dominant disease using the DOMINO software (Quinodoz et al., 2017) deems the gene to be 'very likely recessive'. This is despite the relatively high $\mathrm{pLI}$ of the gene (0.74) and low (0.11) observed/expected ratio in gnomADv2.1.1 (https://doi.org/10.1038/s41586-020-2308-7e). Additionally, homozygous loss of function variants were not seen for PAGR1 gene in gnomADv2.1.1, gnomADv3.1.1, TOPMed and GME variome (Scott et al., 2016) population databases.

The PAGR1 missense variant (c.274A > G; p.Ser92Gly) is localized to a highly conserved region in a Glu-rich domain of unknown 
function. Although the variant is very rare in the general population, it is more common in the Ashkenazi Jewish population, with an allele frequency of 0.00173 (https://doi.org/10.1038/s41586-020-2308-7), and no reported homozygous individuals. This variant was part of a region of homozygosity in the three affected individuals from the two unrelated families (family A - II-2, family B - II-2, II-3), indicating a shared ancestral chromosome with the variant.

Considering all the above, we suggest c.274A > G in PAGR1 is a new candidate Ashkenazi Jewish founder variant for a severe recessive disorder manifested by microcephaly, distal arthrogryposis and developmental delay. We hypothesize that this missense variant might retain some residual function (representing a hypomorphic allele), enabling viability to term. Further functional studies are needed in order elucidate the role of PAGR1 in human diseases.

\section{ACKNOWLEDGMENTS}

We would like to thank the families for participating in this research. Support to WKC was provided by SFARI and the JPB Foundation. Sequencing and analysis were provided by the Broad Institute of MIT and Harvard Center for Mendelian Genomics (Broad CMG) and was funded by the National Human Genome Research Institute, the National Eye Institute, and the National Heart, Lung and Blood Institute grant UM1 HG008900 and in part by National Human Genome Research Institute grant R01 HG009141. We would also like to thank Volkan Okur, Jiangyuan $\mathrm{Hu}$, and Anne O'Donnell Luria for their assistance.

\section{AUTHORS CONTRIBUTION STATEMENT}

All authors listed have made substantial contributions to conception, design, or acquisition of data, analysis and interpretation of data. All have been involved in drafting the manuscript or revising it critically for important intellectual content and given final approval of the version to be published.

All agreed to be accountable for all aspects of the work in ensuring that questions related to the accuracy or integrity of any part of the work are appropriately investigated and resolved.

\section{CONFLICT OF INTEREST}

The authors declare no conflict of interests.

\section{DATA AVAILABILITY STATEMENT}

The data is available upon request by contacting the authors. Otherwise the variant discussed was deposited in the ClinVar database as a likely pathogenic variant (accession number SCV001733594.1).

\section{ORCID}

Hagit Daum (D) https://orcid.org/0000-0002-8318-3599

\section{REFERENCES}

Abu-Libdeh, B., Mor-Shaked, H., Atawna, A. A., Gillis, D., Halstuk, O., Shaul-Lotan, N., Slae, M., Sultan, M., Meiner, V., Elpeleg, O., \& Harel, T. (2021). Homozygous variant in MADD, encoding a Rab guanine nucleotide exchange factor, results in pleiotropic effects and a multisystemic disorder. European Journal of Human Genetics, 29(6), 977-987.

Al-Jawahiri, R., Jones, M., \& Milne, E. (2019). Atypical neural variability in carriers of $16 \mathrm{p} 11.2$ copy number variants. Autism Research, 12(9), 1322-1333.

Birnbaum, R., Jaffe, A. E., Hyde, T. M., Kleinman, J. E., \& Weinberger, D. R. (2014). Prenatal expression patterns of genes associated with neuropsychiatric disorders. The American Journal of Psychiatry, 171(7), 758-767.

Blaker-Lee, A., Gupta, S., McCammon, J. M., De Rienzo, G., \& Sive, H. (2012). Zebrafish homologs of genes within 16p11.2, a genomic region associated with brain disorders, are active during brain development, and include two deletion dosage sensor genes. Disease Models \& Mechanisms, 5(6), 834-851.

Cho, Y. W., Hong, T., Hong, S., Guo, H., Yu, H., Kim, D., Guszczynski, T., Dressler, G. R., Copeland, T. D., Kalkum, M., \& Ge, K. (2007). PTIP associates with MLL3- and MLL4-containing histone H3 lysine 4 methyltransferase complex. The Journal of Biological Chemistry, 282(28), 20395-20406

Dahary, D., Golan, Y., Mazor, Y., Zelig, O., Barshir, R., Twik, M., Iny Stein, T., Rosner, G., Kariv, R., Chen, F., Zhang, Q., Shen, Y., Safran, M., Lancet, D., \& Fishilevich, S. (2019). Genome analysis and knowledgedriven variant interpretation with TGex. BMC Medical Genomics, 12(1), 200.

Ekstein, J., \& Katzenstein, H. (2001). The Dor Yeshorim story: communitybased carrier screening for Tay-Sachs disease. Advances in Genetics, 44, 297-310.

Gong, Z., Cho, Y. W., Kim, J. E., Ge, K., \& Chen, J. (2009). Accumulation of Pax2 transactivation domain interaction protein (PTIP) at sites of DNA breaks via RNF8-dependent pathway is required for cell survival after DNA damage. The Journal of Biological Chemistry, 284(11), 7284-7293.

Jenkins, J., Chow, V., Blaskey, L., Kuschner, E., Qasmieh, S., Gaetz, L., Edgar, J. C., Mukherjee, P., Buckner, R., Nagarajan, S. S., Chung, W. K., Spiro, J. E., Sherr, E. H., Berman, J. I., \& Roberts, T. P. (2016). Auditory Evoked M100 Response Latency is Delayed in Children with $16 \mathrm{p} 11.2$ Deletion but not 16p11.2 Duplication. Cerebral Cortex, 26(5), 19571964.

Kim, S. H., Green-Snyder, L., Lord, C., Bishop, S., Steinman, K. J., Bernier, R., Hanson, E., Goin-Kochel, R. P., \& Chung, W. K. (2020). Language characterization in 16p11.2 deletion and duplication syndromes. American Journal of Medical Genetics. Part B, Neuropsychiatric Genetics, 183(6), 380-391.

Kumar, A., Lualdi, M., Loncarek, J., Cho, Y. W., Lee, J. E., Ge, K., \& Kuehn, M. R. (2014). Loss of function of mouse Pax-Interacting Protein 1-associated glutamate rich protein 1a (Pagr1a) leads to reduced Bmp2 expression and defects in chorion and amnion development. Developmental Dynamics, 243(7), 937-947.

Lee, J. E., Cho, Y. W., Deng, C. X., \& Ge, K. (2020). MLL3/MLL4-Associated PAGR1 Regulates Adipogenesis by Controlling Induction of C/EBP $\beta$ and C/EBPS. Molecular and Cellular Biology, 40(17), 209-220.

Liang, J., Zhang, H., Zhang, Y., \& Shang, Y. (2009). GAS, a new glutamaterich protein, interacts differentially with SRCs and is involved in oestrogen receptor function. EMBO Reports, 10(1), 51-57.

Morson, S., Yang, Y., Price, D. J., \& Pratt, T. (2021). Expression of Genes in the 16p11.2 Locus during Development of the Human Fetal Cerebral Cortex. Cereb Cortex, 31(9), 4038-4052.

Niarchou, M., Chawner, S. J. R. A., Doherty, J. L., Maillard, A. M., Jacquemont, S., Chung, W. K., Green-Snyder, L., Bernier, R. A., GoinKochel, R. P., Hanson, E., Linden, D. E. J., Linden, S. C., Raymond, F. L., Skuse, D., Hall, J., Owen, M. J., \& Bree, M. B. M. V. (2019). Psychiatric disorders in children with 16p11.2 deletion and duplication. Transl Psychiatry, 9(1), 8. 
Okur, V., LeDuc, C. A., Guzman, E., Valivullah, Z. M., Anyane-Yeboa, K., \& Chung, W. K. (2019). Homozygous noncanonical splice variant in. Cold Spring Harb Mol Case Stud, 5(3), a004101.

Quinodoz, M., Royer-Bertrand, B., Cisarova, K., Di Gioia, S. A., SupertiFurga, A., \& Rivolta, C. (2017). DOMINO: Using Machine Learning to Predict Genes Associated with Dominant Disorders. American Journal of Human Genetics, 101(4), 623-629.

Retterer, K., Juusola, J., Cho, M. T., Vitazka, P., Millan, F., Gibellini, F., Vertino-Bell, A., Smaoui, N., Neidich, J., Monaghan, K. G., McKnight, D., Bai, R., Suchy, S., Friedman, B., Tahiliani, J., PinedaAlvarez, D., Richard, G., Brandt, T., Haverfield, E., ... Bale, S. (2016) Clinical application of whole-exome sequencing across clinical indications. Genetics in Medicine, 18(7), 696-704.

Scott, E. M., Halees, A., Itan, Y., Spencer, E. G., He, Y., Azab, M. A., Gabriel, S. B., Belkadi, A., Boisson, B., Abel, L., Clark, A. G., Greater Middle East Variome Consortium, Alkuraya, F. S., Casanova, J. L., \& Gleeson, J. G. (2016 Sep). Characterization of Greater Middle Eastern genetic variation for enhanced disease gene discovery. Nature Genetics, 48(9), 1071-1076.

Shinawi, M., Liu, P., Kang, S. H., Shen, J., Belmont, J. W., Scott, D. A., Probst, F. J., Craigen, W. J., Graham, B. H., Pursley, A., Clark, G., Lee, J., Proud, M., Stocco, A., Rodriguez, D. L., Kozel, B. A., Sparagana, S., Roeder, E. R., McGrew, S. G., ... Lupski, J. R. (2010). Recurrent reciprocal 16p11.2 rearrangements associated with global developmental delay, behavioural problems, dysmorphism, epilepsy, and abnormal head size. Journal of Medical Genetics, 47(5), 332-341.

Sobreira, N., Schiettecatte, F., Valle, D., \& Hamosh, A. (2015). GeneMatcher: a matching tool for connecting investigators with an interest in the same gene. Human Mutation, 36(10), 928-930.

Steinman, K. J., Spence, S. J., Ramocki, M. B., Proud, M. B., Kessler, S. K., Marco, E. J., Green Snyder, L., D'Angelo, D., Chen, Q., Chung, W. K.
Sherr, E. H., \& Consortium, S. V. (2016). 16p11.2 deletion and duplication: Characterizing neurologic phenotypes in a large clinically ascertained cohort. American Journal of Medical Genetics. Part A, 170(11), 2943-2955.

Stelzer G, Plaschkes I, Oz-Levi D, Alkelai A, Olender T, Zimmerman S, Twik M, Belinky F, Fishilevich S, Nudel R, Guan-Golan Y, Warshawsky D, Dahary D, Kohn A, Mazor Y, Kaplan S, Iny Stein T, Baris HN, Rappaport N, Safran M, Lancet D. 2016. VarElect: the phenotype-based variation prioritizer of the GeneCards Suite. BMC Genomics 17, 444.

Zhang, Z., Sun, Y., Cho, Y. W., Chow, C. C., \& Simons, S. S. (2013). PA1 protein, a new competitive decelerator acting at more than one step to impede glucocorticoid receptor-mediated transactivation. The Journal of Biological Chemistry, 288(1), 42-58.

\section{SUPPORTING INFORMATION}

Additional supporting information may be found in the online version of the article at the publisher's website.

How to cite this article: Daum, H., Ganapathi, M., Hirsch, Y., Griffin, E. L., LeDuc, C. A., Hagen, J., Yagel, S., Meiner, V., Chung, W. K., \& Mor-Shaked, H. (2021). Bi-allelic PAGR1 variants are associated with microcephaly and a severe neurodevelopmental disorder: Genetic evidence from two families. American Journal of Medical Genetics Part A, 1-7. https://doi.org/10.1002/ajmg.a.62513 